\title{
Use of Quantum Mechanical Methods to Obtain a Bohm-Type Coefficient of Diffusion
}

\author{
H. JiméneZ-Domínguez* \\ Instituto Nacional de Investigaciones Nucleares \\ Apartado Postal No. 18-1027, Col. Escandón, México 11801, D.F. México
}

(Received January 15, 2009; in final form May 29, 2009)

\begin{abstract}
A Bohm-type coefficient of diffusion is obtained by means of a procedure starting from the Hamiltonian of a single electron in a dilute plasma in the presence of an external, uniform magnetic field of constant magnitude and an electrical potential that serves to simulate an electrical fluctuation which drives the guiding center drift. Using the concept of the differentiation of operators with respect to time, the formula for the well-known $\boldsymbol{E} \times \boldsymbol{B}$ drift velocity is recovered. Finally, the solution of a quantum mechanical equation of motion for the guiding center is found in the quasi-classical approximation to obtain a diffusion coefficient.
\end{abstract}

PACS numbers: 51.20.+d, 52.20.Dq, 52.25.Fi, 52.25.Xz

\section{Introduction}

The phenomenon of the diffusion of plasma across a magnetic field has been observed in many natural and laboratory plasmas. In particular, the Bohm diffusion is important in some plasma experiments, the corresponding coefficient being originally introduced semi-empirically by Bohm in 1946 [1]. Later experimental observations in stellarators [2] prompted Spitzer [3] to propose in 1960 a mechanism to explain the appearance of this diffusion phenomenon as well as a way of deriving the corresponding coefficient by analyzing the mean square value of the particle displacements due to $\boldsymbol{E} \times \boldsymbol{B}$ drifts generated by electric field fluctuations. By means of this approach, Spitzer found an expression which is proportional to the coefficient introduced by Bohm.

Various mechanisms have been proposed to explain the existence of the Bohm diffusion [4] and in Ref. [5] the authors propose that this coefficient may have a quantum mechanical origin. The present paper is based on the idea that a Bohm-type diffusion coefficient could be obtained by using a quantum mechanical approach. The following are the main reasons which have led the present author to pursue this task. It is well known that the Bohm diffusion coefficient presents two important features that distinguish it from the "classical" diffusion coefficients [6], viz. (a) its non-dependence on the particle density and (b) its $1 / B$ dependence. The former aspect may suggest that a Bohm-type diffusion coefficient could be obtained by studying the behavior of the motion of a single

\footnotetext{
* e-mail: homero.jimenez@inin.gob.mx
}

electron in a dilute plasma in the presence of an external, uniform magnetic field of constant magnitude and an electrical potential. The latter aspect could be related to the quantum mechanical feature that the position of the guiding center of a magnetically gyrating charged particle is uncertain and is located inside an area of uncertainty which is inversely proportional to the magnitude of the magnetic field ([7], p. 756, [8]). It is then the purpose of this paper to study, over a time comparable with a cyclotron period $T_{\mathrm{c}}$, the behavior of a single electron with a thermal energy $k T$ in a dilute plasma (to avoid considering charge particle interactions) in the presence of an external, uniform magnetic field of constant magnitude from a quantum mechanical point of view, starting from a Hamiltonian that includes an electrical potential that serves to simulate an electrical fluctuation which drives the guiding center drift.

\section{Analytic calculations}

Let us consider the problem of a moving electron with charge $e$ and mass $m$ in the presence of a constant, uniform magnetic field $\boldsymbol{B}$ which for convenience will be along the positive direction of the $z$ axis. In this circumstance, the components of the vector potential can be conveniently written for the purpose of this paper as follows:

$$
A_{x}=-\frac{1}{2} B y, \quad A_{y}=\frac{1}{2} B x, \quad A_{z}=0 .
$$

As is well known from classical physics, the electron moves along a well-defined circular orbit with a well-defined guiding center and a frequency $\omega_{\mathrm{c}}=e B / m c$ called the cyclotron frequency and a radius $\rho_{\mathrm{L}}=\nu_{\mathrm{th}} / \omega_{\mathrm{c}}$ 
called the Larmor radius, in which $\nu_{\text {th }}$ is the thermal velocity of the electron.

Let us now write down the Hamiltonian operator for a single electron with a thermal energy $k T$ in a dilute plasma in the presence of the magnetic field corresponding to the vector potential (1) and an electrical potential $V(x, y)$ to describe the motion on the $x y$ plane. This potential will be assumed to vary slowly in times longer than a cyclotron period and over distances more extensive than the Larmor radius in such a way that the resulting drift velocity of the guiding center changes very slowly in time and space. The interactions between charged particles will not be taken into account in the present treatment. The motion along the field lines will not be considered in this paper and the term corresponding to the spin of the electron will not be taken into account in the Hamiltonian. With these considerations, the Hamiltonian is written in terms of components of the vector potential given by (1) as follows:

$$
\begin{aligned}
\hat{H} & =\frac{1}{2 m}\left(\hat{p}_{x}+\frac{e B y}{2 c}\right)^{2}+\frac{1}{2 m}\left(\hat{p}_{y}-\frac{e B x}{2 c}\right)^{2} \\
& +e V(x, y) \equiv \hat{H}_{B}+\hat{H}_{E},
\end{aligned}
$$

where $\hat{p}_{x}=-\mathrm{i} \hbar \frac{\partial}{\partial x}$ and $\hat{p}_{y}=-\mathrm{i} \hbar \frac{\partial}{\partial y}$ are the operators of the $x$ and $y$ components of the canonical momentum and $\hat{H}_{B}$ and $\hat{H}_{E}$ stand for magnetic and electric Hamiltonian, respectively. As is well known ([7], pp. 745-746), the magnetic part of this Hamiltonian can be written as the sum of a Hamiltonian for the two-dimensional harmonic oscillator $\hat{H}_{x y}$ plus a term which is proportional to the angular momentum operator $\hat{L}_{z}$ corresponding to the motion of the electron along its circular orbit on the $x y$ plane. The model represented by the Hamiltonian (2) describes a two-dimensional motion of a typical electron in a magnetized dilute plasma in the presence of an electrical potential that serves to simulate an electrical fluctuation that will allow the electron to drift. On the other hand, starting from the classical expressions for the coordinates $\left(x_{0}, y_{0}\right)$ of the guiding center of the orbit of the electron, given in Ref. [9] and considering the vector potential (1), the corresponding operator coordinates of the guiding center $\left(\hat{x}_{0}, \hat{y}_{0}\right)$ can be written as follows:

$$
\begin{aligned}
& \hat{x}_{0}=\hat{x}+\frac{c}{e B}\left(\hat{p}_{y}-\frac{e B}{2 c} \hat{x}\right), \\
& \hat{y}_{0}=\hat{y}-\frac{c}{e B}\left(\hat{p}_{x}+\frac{e B}{2 c} \hat{y}\right),
\end{aligned}
$$

where $\hat{x}, \hat{y}$ are the operator coordinates of the electron. As can easily be verified, the operator coordinates of the guiding center commute with $\hat{H}_{B}$ so that the physical quantities $x_{0}, y_{0}$ are constants of the motion for the magnetic Hamiltonian, indeed,

$$
\hat{\dot{x}}_{0}=\frac{\mathrm{i}}{\hbar}\left[\hat{H}_{B}, \hat{x}_{0}\right]=0, \quad \hat{\dot{y}}_{0}=\frac{\mathrm{i}}{\hbar}\left[\hat{H}_{B}, \hat{y}_{0}\right]=0,
$$

where the dot represents the derivative with respect to time. Equations (4) mean that the guiding center does not move in the absence of an electrical potential. On the other hand, as can also be easily shown, the operator coordinates of the guiding center do not commute with each other; in fact, their commutator is

$$
\left[\hat{x}_{0}, \hat{y}_{0}\right]=-\mathrm{i} \frac{\hbar c}{e B} \text {. }
$$

Therefore, the following uncertainty principle can be constructed:

$$
\Delta x_{0} \Delta y_{0} \geq \frac{1}{2} \frac{\hbar c}{e B} .
$$

Expression (6) indicates that the position of the guiding center is uncertain inside a region of linear dimensions

$$
\Delta r_{0} \approx \sqrt{\hbar c / e B}
$$

The orbit itself is also "spread out" to an extent of the same order of magnitude in the radial direction [8]. Expressions (6), (7) indicate that the size of the uncertainty area of the guiding center is inversely proportional to the intensity of the magnetic field and does not depend upon the mass of the electron.

For future reference, it is convenient to write down the ratio $\left(\rho_{\mathrm{L}} / \Delta r_{0}\right)^{2}$ between the squares of the Larmor radius and the linear dimensions of the uncertainty area given by Eq. (7). In many experiments the magnitude of this ratio is very large and can be written as

$$
\left(\frac{\rho_{\mathrm{L}}}{\Delta r_{0}}\right)^{2} \approx \frac{k T}{\hbar \omega_{\mathrm{c}}} \gg 1 \text {. }
$$

\section{The drift velocity}

As in Eqs. (4), let us find the operators of the time derivatives of the physical quantities $x_{0}, y_{0}$ but now using the complete Hamiltonian (2). After some algebra and considering that the operators $\hat{x}_{0}$ and $\hat{y}_{0}$ commute with $\hat{H}_{B}$, the following results are obtained:

$$
\begin{aligned}
& \hat{\dot{x}}_{0}=\frac{\mathrm{i}}{\hbar}\left[\hat{H}, \hat{x}_{0}\right]=-\frac{c}{B} \frac{\partial V(x, y)}{\partial y}=c \frac{E_{y}}{B} \equiv u_{D x}, \\
& \hat{\dot{y}}_{0}=\frac{\mathrm{i}}{\hbar}\left[\hat{H}, \hat{y}_{0}\right]=\frac{c}{B} \frac{\partial V(x, y)}{\partial x}=-c \frac{E_{x}}{B} \equiv u_{D y},
\end{aligned}
$$

which are the well-known components of the drift velocity of the guiding center of the electron's orbit in the presence of a constant magnetic field $B$ and the electric field related to the electrical potential $V(x, y)$.

\section{Obtaining a Bohm-type coefficient of diffusion}

In this section, work will be carried out to describe the motion of the guiding center on which both the charge and the mass of the electron will be assumed to be sited. It will be enough to consider only the motion along the $x$ coordinate to have an idea of the nature of the diffusion process of the guiding center as well as an estimation of the value of the corresponding coefficient. Therefore, let us start from the following expression of type (4) for the product $x_{0} \dot{x}_{0}$ using the complete Hamiltonian (2):

$$
\left(\frac{\mathrm{d} x_{0} \dot{x}_{0}}{\mathrm{~d} t}\right)_{\text {operator }}=\frac{\mathrm{i}}{\hbar}\left[\hat{H}, \hat{x}_{0} \hat{\dot{x}}_{0}\right] .
$$

A similar equation for the $y$ component of the position vector of the guiding center may be written. Consider now that the electric Hamiltonian $\hat{H}_{E}=e V(x, y)$ 
commutes with $\hat{\dot{x}}_{0}=-\frac{c}{B} \frac{\partial V(x, y)}{\partial y}$ and that the following expression is valid:

$$
\begin{aligned}
& {\left[\hat{H}_{B}, \hat{x}_{0} \hat{\dot{x}}_{0}\right]=\hat{x}_{0}\left[\hat{H}_{B}, \hat{\dot{x}}_{0}\right]+\left[\hat{H}_{B}, \hat{x}_{0}\right] \hat{\dot{x}}_{0}} \\
& \quad=\hat{x}_{0}\left[\hat{H}_{B}, \hat{\dot{x}}_{0}\right]
\end{aligned}
$$

as $\hat{H}_{B}$ and $\hat{x}_{0}$ commute with each other. Therefore, Eq. (11) is rewritten as

$$
\frac{\mathrm{d}}{\mathrm{d} t} x_{0} \dot{x}_{0}=\dot{x}_{0}^{2}+\frac{\mathrm{i}}{\hbar}\left[H_{\mathrm{B}}, x_{0} \dot{x}_{0}\right] \text {, }
$$

where the operator caps have been eliminated, as will be done from now on. On the other hand, the Hamiltonian $H_{B}$ can also be written as ([7], p. 754):

$$
H_{\mathrm{B}}=\hbar \omega_{\mathrm{c}}\left(N_{\mathrm{d}}+\frac{1}{2}\right),
$$

in which the eigenvalues of the dimensionless number operator $N_{\mathrm{d}}$ are the positive integers and zero. Substitution of (13) in (12) produces the expression

$$
\frac{\mathrm{d}}{\mathrm{d} t} x_{0} \dot{x}_{0}=\dot{x}_{0}^{2}+\mathrm{i} \omega_{\mathrm{c}}\left[N_{\mathrm{d}}, x_{0} \dot{x}_{0}\right] \text {, }
$$

which after performing the commutator operation gives the operator equation

$$
\frac{\mathrm{d}}{\mathrm{d} t} x_{0} \dot{x}_{0}=\dot{x}_{0}^{2}+\mathrm{i} \omega_{\mathrm{c}} N_{\mathrm{d}} x_{0} \dot{x}_{0} \text {. }
$$

However, just as in the case of expression (8), it also turns out that the following expression holds:

$$
\frac{\rho_{\mathrm{L}}}{\lambda_{\mathrm{B}}} \approx \frac{k T}{\hbar \omega_{\mathrm{c}}} \gg 1
$$

where $\lambda_{\mathrm{B}}$ is the de Broglie wavelength of the orbiting electron. The last expression suggests that the system can be treated by means of a quasi-classical approach, in which case it is possible to use the mean values of observables of the harmonic oscillator in terms of quasi-classical states, as given in Ref. [7], p. 740. Therefore, taking the mean value on both sides of expression (14) considering that $N_{\mathrm{d}}$ and the product $x_{0} \dot{x}_{0}$ are non-correlated quantities, the following equation is obtained:

$$
\frac{\mathrm{d}}{\mathrm{d} t}\left\langle x_{0} \dot{x}_{0}\right\rangle=\left\langle\dot{x}_{0}^{2}\right\rangle+\mathrm{i} \Omega\left\langle x_{0} \dot{x}_{0}\right\rangle,
$$

where

$$
\Omega \equiv \omega_{\mathrm{c}}\left\langle N_{\mathrm{d}}\right\rangle .
$$

The value of $\left\langle N_{\mathrm{d}}\right\rangle$ for an electron with thermal energy $k T$ and with oscillator energy $\hbar \omega_{\mathrm{c}}$ can be found by using Eq. (79) of Ref. [7], p. 740. This value is

$$
\left\langle N_{\mathrm{d}}\right\rangle=\frac{k T}{\hbar \omega_{\mathrm{c}}}
$$

which, as seen in Eqs. (8), (15), is a rather large quantity. At this point it is convenient to notice that Eq. (18) can be rewritten as the ratio of two quantities, each of which has the dimensions of a diffusion coefficient

$$
\left\langle N_{\mathrm{d}}\right\rangle=\frac{c k T / q B}{\hbar / m},
$$

which, as before, should be a very large quantity. It is noticed here that the numerator is a Bohm-type coefficient of diffusion and the denominator is a "quantum coeffi- cient of diffusion". Expression (19) will be interpreted in the sense that the guiding center diffuses simultaneously with those two coefficients of diffusion, the step size of each one being deduced further below.

Assuming that the guiding center starts out at $t=0$ at the position $x_{0}=0$ and that $\left\langle\dot{x}_{0}^{2}\right\rangle$ changes very slowly in time, in agreement with the assumption made before, so that it can be taken out of the time integration, the general solution of Eq. (16) is written as

$$
\left\langle x_{0} \dot{x}_{0}\right\rangle=\frac{\left\langle\dot{x}_{0}^{2}\right\rangle}{\mathrm{i} \Omega}\left[\mathrm{e}^{\mathrm{i} \Omega t}-1\right] \text {. }
$$

Considering that the operations of taking both the time derivative and the mean value of a physical quantity commute [10], the left hand side of Eq. (20) can be written as $\left\langle x_{0} \dot{x}_{0}\right\rangle=\frac{1}{2} \frac{\mathrm{d}}{\mathrm{d} t}\left\langle x_{0}^{2}\right\rangle$; therefore, Eq. (20) becomes

$$
\frac{\mathrm{d}}{\mathrm{d} t}\left\langle x_{0}^{2}\right\rangle=\frac{2\left\langle\dot{x}_{0}^{2}\right\rangle}{\mathrm{i} \Omega}\left[\mathrm{e}^{\mathrm{i} \Omega t}-1\right] .
$$

Integrating this equation using the same conditions as in the integration of Eq. (16), the following expression is obtained:

$$
\left\langle x_{0}^{2}\right\rangle=-\frac{2\left\langle\dot{x}_{0}^{2}\right\rangle}{\Omega^{2}}\left[\mathrm{e}^{\mathrm{i} \Omega t}-1-\mathrm{i} \Omega t\right] .
$$

It can be noticed, incidentally, that if $\Omega$ and $\left\langle\dot{x}_{0}^{2}\right\rangle$ in Eq. (22) are written as $\mathrm{i} \gamma$ and $k T / m$, respectively, the form of Eq. (15.6.8) in Ref. [11] is recovered, although this last expression is the solution of the Langevin equation for the motion of a Brownian particle.

Equation (22) can be rewritten as

$$
\left\langle x_{0}^{2}\right\rangle=\left\langle\dot{x}_{0}^{2}\right\rangle t^{2} f(\theta)
$$

where the function $f(\theta)$ is given by the expression

$$
f(\theta)=\frac{\sin ^{2} \theta}{\theta^{2}}+\mathrm{i}\left(\frac{1}{\theta}-\frac{\sin 2 \theta}{2 \theta^{2}}\right),
$$

where $\theta=\frac{\Omega t}{2}$.

Four facts may be readily noted from Eqs. (23) and (24), viz.:

1. Expression (24) is such that $f(-\theta)=f^{*}(\theta)$, which means that its Fourier transform is real; the star stands for "complex-conjugated". This fact gives the character of observable quantities, to the real and imaginary parts of $\left\langle x_{0}^{2}\right\rangle$ in Eq. (23).

2. The real and imaginary parts of Eq. (24) are mutually orthogonal.

3. The real and imaginary parts of Eq. (24) are the Hilbert transform of each other [12].

4. The real and imaginary parts of Eq. (24) fulfill the Parseval relation, as it should be for pairs of expressions that are related by the Hilbert transform.

As a consequence of these facts, expression (23) may be considered as a dispersion relation in the time domain where the real and imaginary parts, both observable, play the emissive and dispersive (in this case diffusive) roles, respectively. Figure 1 depicts the real and imaginary 


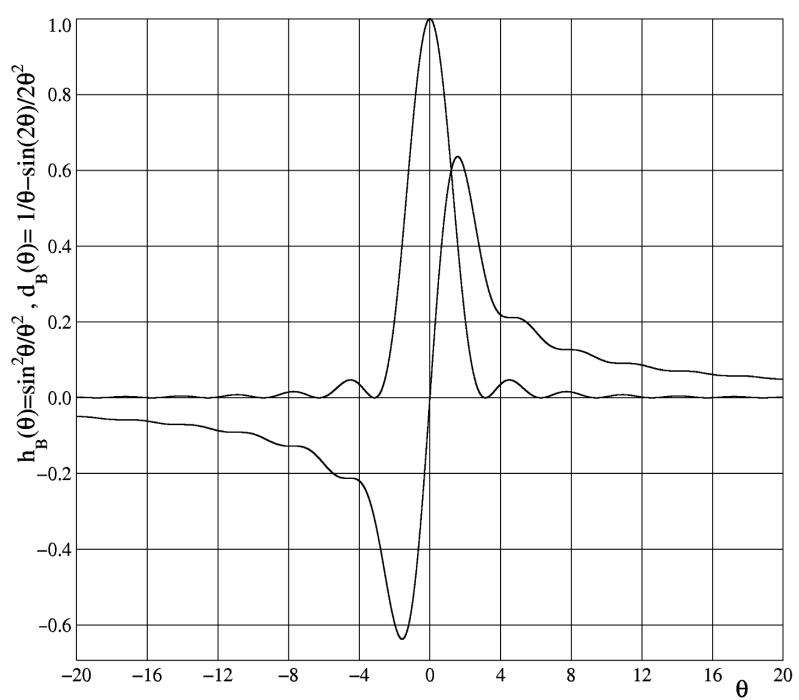

Fig. 1. Real $\left(h_{B}(\theta)\right)$ and imaginary $\left(d_{B}(\theta)\right)$ parts of the function $f(\theta)$ given by Eq. (24) in text.

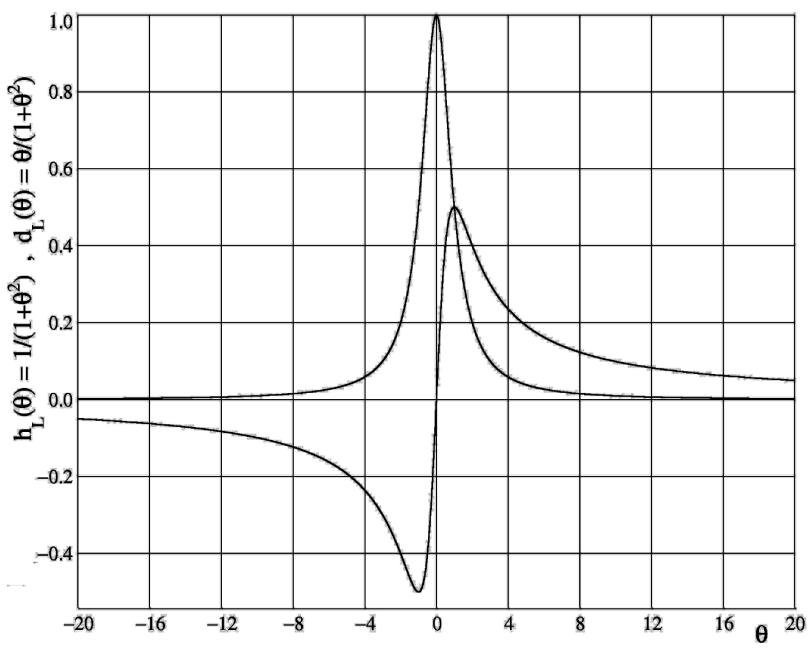

Fig. 2. Real $\left(h_{\mathrm{L}}(\theta)\right)$ and imaginary $\left(d_{\mathrm{L}}(\theta)\right)$ parts of the function $f_{\mathrm{L}}(\theta)$ given by Eq. (25) in text.

parts of the expression $f(\theta)$ given by Eq. (24), as functions of $\theta$. For comparison purposes let us introduce the Lorentzian dispersion relation $f_{\mathrm{L}}(\theta)$ given by

$$
f_{\mathrm{L}}(\theta)=\frac{1}{1+\theta^{2}}+\mathrm{i} \frac{\theta}{1+\theta^{2}} .
$$

In this expression the real and imaginary parts are the Hilbert transform of each other, physically representing an absorptive-dispersive phenomenon in which the absorption part has a Lorentzian shape; Fig. 2 shows the real and imaginary parts of Eq. (25).

Figure 3 depicts two DISPA (DISPersion-Absorption) plots $[13,14]$ in which the real (imaginary) part is represented on the horizontal (vertical) axis; plot B corresponds to Eq. (24) and for comparison plot L is drawn, corresponding to the Lorentzian dispersion relation given

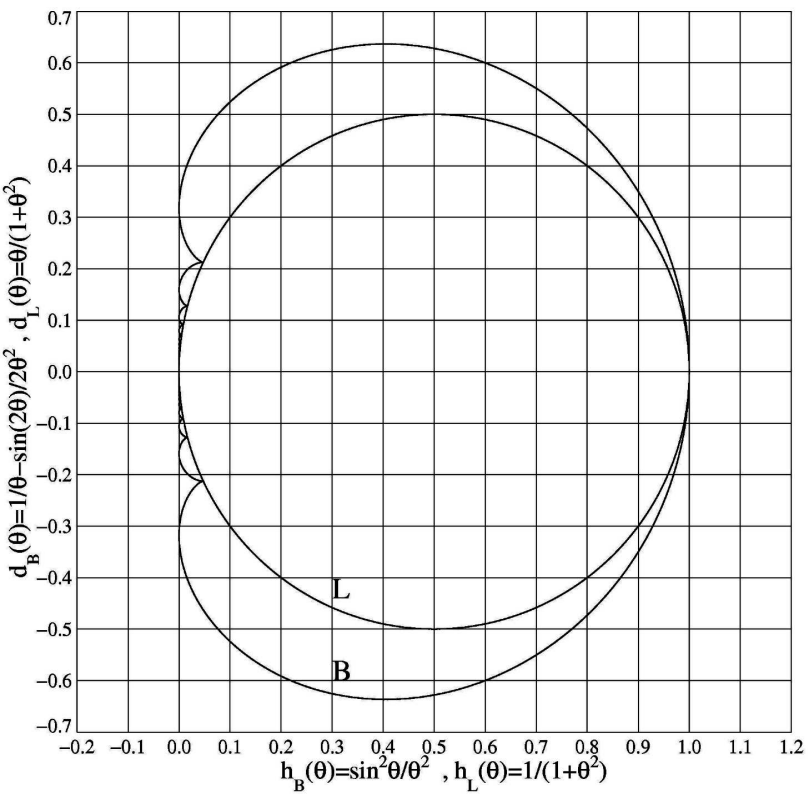

Fig. 3. DISPA plots of Eq. (24) (curve B) and Eq. (25) (curve L). DISPA plot of Eq. (25) is a circumference of radius $1 / 2$.

by Eq. (25). The DISPA plot of Eq. (25) is a circumference of radius $1 / 2$. It is observed from Fig. 3 that plot B represents a "spectrum" that is a broadened version of the one represented by plot L (see Refs. [13, 14]); this can also be visualized in Figs. 1, 2.

It is convenient to note here that the ratio $k T / \hbar \omega_{\mathrm{c}}$ (see Eq. (18)) is so large that the variable $\theta=\Omega t / 2=$ $\left(\left\langle N_{\mathrm{d}}\right\rangle \omega_{\mathrm{c}} t\right) / 2$ spans every interesting feature in Figs. 1, 3 for a wide interval of time, in particular for $0 \leq t \leq T_{\mathrm{c}}$ which is the interval of interest in this paper, as was mentioned in the introduction.

By looking at the symmetrical plot B in Fig. 3 from right to left, an interesting feature to notice is that most of the pattern belongs to a very small interval of time; for example, the first zero of the function $\sin ^{2} \theta / \theta^{2}$, which appears at $\theta \approx 3.15$, corresponds to a time $t \sim 6.3 /\left\langle N_{\mathrm{d}}\right\rangle \omega_{\mathrm{c}}$, which is a very small quantity; this corresponds to the uppermost point at which plot B touches the vertical axis in Fig. 3. Only the smaller wavy structure of plot $\mathrm{B}$ represents the diffusion phenomenon for longer times, $t \sim T_{\mathrm{c}}$, as will be seen below.

In the following, the behavior of the real and imaginary parts of Eq. (23) will be analyzed. The real part is

$$
\operatorname{Re}\left\langle x_{0}^{2}\right\rangle=\left\langle\dot{x}_{0}^{2}\right\rangle t^{2} \frac{\sin ^{2} \theta}{\theta^{2}} .
$$

For $\theta \ll 1$ this expression reduces to

$$
\operatorname{Re}\left\langle x_{0}^{2}\right\rangle=\left\langle\dot{x}_{0}^{2}\right\rangle t^{2}
$$

which means that the guiding center moves freely during a very short initial interval of time. The right hand side of Eq. (27) agrees with the result of the usual analysis of the mean square displacement of a Brownian particle by means of the Langevin equation [11]. 
Due to the behavior of the function $\sin ^{2} \theta / \theta^{2}$ for $\theta \gg 1$, see Fig. 1, Eq. (26) becomes

$$
\operatorname{Re}\left\langle x_{0}^{2}\right\rangle \approx 0 .
$$

In other words, the real part of Eq. (23) has importance only during a very short initial interval of time.

In order to handle the imaginary part of expression (23), i.e.

$$
\operatorname{Im}\left\langle x_{0}^{2}\right\rangle=\left\langle\dot{x}_{0}^{2}\right\rangle t^{2}\left(\frac{1}{\theta}-\frac{\sin 2 \theta}{2 \theta^{2}}\right),
$$

it is necessary to go back to Eq. (20), which after some treatment will serve to eliminate the factor $\left\langle\dot{x}_{0}^{2}\right\rangle$ from Eq. (29) in order to get an expression from which a diffusion coefficient can be obtained. Accordingly, let us first write Eq. (20) as a dimensionless expression in order to get the probability of finding the system in a given state of motion at time $t$. To do this, first let us multiply both members by the electron mass $m$, which was assumed to be located at the guiding center. Thus, an expression with the dimensions of an action is obtained. Then by dividing this expression by $\hbar$, a dimensionless quantity is obtained. Finally, let us take the square of the absolute value on both sides. The result is the following:

$$
\left|\frac{\left\langle x_{0} m \dot{x}_{0}\right\rangle}{\hbar}\right|^{2}=\frac{4\left|\left\langle m \dot{x}_{0}^{2}\right\rangle\right|^{2} \sin ^{2} \frac{\Omega t}{2}}{\hbar^{2} \Omega^{2}} .
$$

The right hand side of Eq. (30) has the form of the expression obtained in first order perturbation theory for the quantum mechanical probability of finding a system in a given state [15]; in the present case, it is the probability of finding the guiding center in the state of motion $m \dot{x}_{0}^{2}$ at time $t$ under the action of the electric field produced by the potential $V(x, y)$ on the location of the guiding center where the electron mass and charge were assumed to be located.

Considering the comments made before concerning plot $\mathrm{B}$ in Fig. 3, the time average of the right hand side of Eq. (30) over a cyclotron period $T_{\mathrm{c}}$ is taken and made equal to one, to indicate the certainty of occurrence of reaching the state of motion $m \dot{x}_{0}^{2}$; the result is

$$
\begin{gathered}
\frac{2\left|\left\langle m \dot{x}_{0}^{2}\right\rangle\right|^{2}}{\hbar^{2} \Omega^{2}}=1 \\
\left\langle\dot{x}_{0}^{2}\right\rangle=\frac{\hbar \Omega}{\sqrt{2} m} .
\end{gathered}
$$

Remembering that the quantity $\dot{x}_{0}$ on the left hand side of this expression represents the drift velocity of the guiding center, before going on with the calculation, this velocity will be written in terms of the electric field generated by the potential $V(x, y)$ in order to compare the resulting expression with Spitzer Eq. (5), Ref. [3]. By doing so and rearranging Eq. (32) using Eq. (17), the following expression for the mean square value of the electric field is obtained:

$$
\left\langle E_{y}^{2}\right\rangle=\frac{k T B^{2}}{\sqrt{2} m c^{2}} .
$$

It is seen that this expression is consistent with Spitzer Eq. (5), Ref. [3], although there the mean square value was taken over a set of particles.

Reassuming the calculation and substituting Eq. (32) in Eq. (29), the following expression is obtained:

$$
\operatorname{Im}\left\langle x_{0}^{2}\right\rangle=\frac{\hbar \Omega}{\sqrt{2} m} t^{2}\left(\frac{1}{\theta}-\frac{\sin 2 \theta}{2 \theta^{2}}\right) .
$$

The right hand side of Eq. (34) tends to zero for $\theta \ll 1$ (see Fig. 1). Therefore, this expression, which is the imaginary part of Eq. (23), is relevant only for large values of $\theta$; indeed, due to the behavior of the functions $(\sin 2 \theta) / 2 \theta^{2}$ and $1 / \theta$ for $\theta \gg 1$, Eq. (34) yields the following expression:

$$
\operatorname{Im}\left\langle x_{0}^{2}\right\rangle=\sqrt{2} \frac{\hbar}{m} t .
$$

If the displacement of the guiding center is considered as a random walk, then the left hand side of Eq. (35) may be written as

$$
\operatorname{Im}\left\langle x_{0}^{2}\right\rangle=2 D t
$$

By comparing Eqs. (35) and (36), the following expression for a quantum coefficient of diffusion for the guiding center is obtained:

$$
D=\frac{1}{\sqrt{2}} \frac{\hbar}{m} .
$$

To estimate the step-size in this diffusion process, a step-time of $\omega_{\mathrm{c}}^{-1}$ will be used in Eq. (34) for $\theta \gg 1$; then substituting $t=\omega_{\mathrm{c}}^{-1}$ there and taking into account that $\left\langle N_{\mathrm{d}}\right\rangle \gg 1$, the following expression is obtained:

$$
\operatorname{Im}\left\langle x_{0}^{2}\right\rangle=\sqrt{2} \frac{\hbar c}{e B} \approx \sqrt{2}\left(\Delta r_{0}\right)^{2},
$$

where the use of Eq. (7) was made. According to Eq. (38), the step-size for quantum diffusion is of the order of the linear dimensions of the uncertainty area of the guiding center (see Eq. (7)). Therefore, the imaginary part of Eq. (23) describes the process of the quantum diffusion of the guiding center with the coefficient given by Eq. (37).

In order to obtain the formula for the mean square displacement for the case of the Bohm-type diffusion, it is necessary to look at Eq. (19) which indicates that the Bohm-type diffusion is $\left\langle N_{\mathrm{d}}\right\rangle$ times as large as the quantum diffusion. It is then expected that the mean square displacement for the Bohm-type diffusion should also be $\left\langle N_{\mathrm{d}}\right\rangle$ times as large as the one for quantum diffusion; hence, the mean square displacement for Bohm-type diffusion is

$$
\left\langle x_{0}^{2}\right\rangle=\left\langle N_{\mathrm{d}}\right\rangle\left\langle\dot{x}_{0}^{2}\right\rangle t^{2} f(\theta) .
$$

From the foregoing work it follows that that the diffusion coefficient should be obtained from the imaginary part of Eq. (39) for $\theta \gg 1$. Using Eq. (32), the following expression is obtained:

$$
\operatorname{Im}\left\langle x_{0}^{2}\right\rangle=\sqrt{2} \frac{c k T}{e B} t .
$$

As before, considering that the motion of the guiding center is a random walk, by combining Eqs. (36) and (40), the following Bohm-type coefficient of diffusion is obtained: 


$$
D=\frac{1}{\sqrt{2}} \frac{c k T}{e B} .
$$

As can be seen, expression (41) is $16 / \sqrt{2}$ times as large as the Bohm diffusion coefficient. To obtain the step-size for this process, use is made of the imaginary part of Eq. (39) which is

$$
\operatorname{Im}\left\langle x_{0}^{2}\right\rangle=\left\langle N_{\mathrm{d}}\right\rangle \frac{\hbar \Omega}{\sqrt{2} m} t^{2}\left(\frac{1}{\theta}-\frac{\sin 2 \theta}{2 \theta^{2}}\right),
$$

where Eq. (32) was substituted in (42). Now, for $\theta \gg 1$ and using $\omega_{\mathrm{c}}^{-1}$ as a step-time, Eq. (42) reduces to

$$
\operatorname{Im}\left\langle x_{0}^{2}\right\rangle=\sqrt{2} \frac{\nu_{\mathrm{th}}^{2}}{\omega_{\mathrm{c}}^{2}}=\sqrt{2} \rho_{\mathrm{L}}^{2}
$$

Then the step size in this diffusion process is of the order of the Larmor radius, i.e. the guiding center passes each time from the original location of its uncertainty area to the location of the orbital uncertainty area provoked by an external electrical fluctuation, thus producing the migration of the guiding center with this step size. The step-size $\rho_{\mathrm{L}}$ is a known characteristic of the Bohm diffusion.

\section{Conclusion}

An elementary quantum mechanical analysis of the motion of the guiding center of a magnetically gyrating plasma electron in the presence of an electrical potential that simulates an electrical fluctuation was carried out in this paper. The drift velocity of the guiding center was first obtained, this information being used to write down a quantum mechanical equation of motion for the product $x_{0} \dot{x}_{0}$. The solution of this equation led to the derivation of two coefficients of diffusion as the imaginary part of the average of the square of the guiding center displacement in terms of quasi-classical states of the harmonic oscillator for the case $\theta \gg 1$. These two coefficients are a "quantum mechanical coefficient of diffusion" and a Bohm-type coefficient of diffusion, which could be interpreted in the sense that the guiding center diffuses simultaneously with those two coefficients, the quantum mechanical coefficient being very small. Therefore, it could be said that the diffusion phenomenon which is considered in this paper has its origin in the quantum mechanical uncertainty of the position of the guiding center as well as in the quantum mechanical "spreading out" of the electron orbit and is provoked by electric field fluctuations. Although the main scaling of the Bohm-type coefficient with the magnetic field and the temperature is that of the well-known Bohm coefficient, it should be mentioned that the model employed here is a very crude one. Specifically, particle interactions which play a paramount role in plasma processes were not taken into account as part of the hy- pothesis and assumptions used in this work. This could explain why one ends up with a coefficient which is $16 / \sqrt{2}$ times as large as the Bohm coefficient. Finally, as the Bohm diffusion still eludes an explanation based on first principles, both the hypothesis and the simple method employed in this paper to obtain a Bohm-type coefficient of diffusion, rendered a result which may encourage the pursuit of further research on this issue.

\section{Acknowledgments}

Thanks are due to Drs. G. López-Laurrabaquio and L. Meléndez-Lugo for valuable comments and for supplying relevant literature. I also thank Dr. J. Klapp and Ing. A. Arenas-Ochoa for graphical art work. Formatting work by Dr. R. Peña-Eguiluz is appreciated.

\section{References}

[1] D. Bohm, E.H.S. Burhop, H.S.W. Massey, R.W. Williams, in: A Study of the Arc Plasma in the Characteristics of Electrical Discharges in Magnetic Fields, Eds. A. Guthrie, R.W. Wakerling, 1st ed., McGraw Hill, 1949, p. 173.

[2] I.A. Bernstein, E.A. Frieman, R.M. Kulsrud, M.N. Rosenbluth, Phys. Fluids 3, 136 (1960).

[3] L. Spitzer Jr., Phys. Fluids 3, 659 (1960).

[4] F.F. Chen, Introduction to Plasma Physics and Controlled Fusion, 2nd ed., Vol. I, Plenum Press, New York 1984, p. 190.

[5] V.S. Belyaev, V.N. Mikhaylov, Laser Phys. II, 957 (2001).

[6] K. Miyamoto, Plasma Physics for Nuclear Fusion, The MIT Press, Massachusetts 1980, p. 199, 219.

[7] C. Cohen-Tannoudji, B. Diu, F. Laloë, Quantum Mechanics, Vol. I, Wiley, New York 1977.

[8] Problems in Quantum Mechanics, Ed. D. terHaar, 3rd ed., Pion Ltd, London 1975, p. 39, 260, Problem 12.

[9] L.D. Landau, E.M. Lifshitz, The Classical Theory of Fields, Pergamon Press, London 1987, p. 53.

[10] L.D. Landau, E.M. Lifshitz, Quantum Mechanics, 2nd ed., Pergamon Press, Addison-Wesley, 1965, Eq. (9.1), p. 26.

[11] F. Reif, Fundamentals of Statistical and Thermal Physics, McGraw-Hill, New York 1965, p. 566.

[12] F.W. King, University of Wisconsin - Eau Claire, fking@uwec.edu, private communication.

[13] A.G. Marshall, D.C. Roe, Anal. Chem. 50, 756 (1978).

[14] D.C. Roe, A.G. Marshall, Anal. Chem. 50, 764 (1978).

[15] L.I. Schiff, Quantum Mechanics, 3rd ed., Mc Graw-Hill, New York 1968, p. 282. 